\title{
Decodificação Iterativa para Concatenação Serial com Codificador Wavelet
}

\author{
Luiz G. de Q. Silveira Júnior, Luiz Felipe de Q. Silveira, Francisco M. de Assis e Ernesto L. Pinto
}

\begin{abstract}
Resumo-Propõe-se neste artigo um esquema de decodificação iterativa para concatenações seriais em que um codificador wavelet é usado como codificador interno. Em particular, apresenta-se uma estrutura original de decodificador wavelet do tipo SISO, com base numa técnica de demodulação com saída suave recentemente proposta para aplicação em sistemas de transmissão sem fio que empregam codificação wavelet. Alguns resultados preliminares de avaliação de desempenho são apresentados, considerando-se uma concatenação com codificador convolucional em canal com desvanecimento plano. Estes resultados mostram a eficácia da técnica de decodificação proposta e indicam que o seu emprego pode levar a novas alternativas para explorar o potencial da codificação wavelet em sistemas de comunicações com desvanecimento.
\end{abstract}

Palavras-Chave-codificação wavelet, decodificação iterativa, desvanecimento Rayleigh, decodificador SISO, informação extrínseca.

Abstract-An iterative decoder for serial concatenations that have a wavelet encoder as the inner encoder is presented in this article. In particular, a novel soft-input soft-output wavelet decoder is proposed, which is based on a soft demodulation mapping recently proposed for wavelet-coded wireless communication systems. Preliminary results of performance evaluation are presented, where a concatenation with a convolutional encoder over a flat-fading channel is investigated. These results show the effectiveness of the proposed decoding technique and suggest that its is potentially useful as a new means to increase the performance benefits produced by wavelet coding in flat fading channels.

Keywords - wavelet coding, iterative decoding, Rayleigh fading channels, SISO decoder, extrinsic information.

\section{INTRODUÇÃO}

A transmissão em canais com desvanecimento variante no tempo continua a ser uma área de grande interesse para o desenvolvimento de novos sistemas de comunicações, e este interesse só tende a aumentar com o surgimento de novos serviços de comunicações para sistemas de transmissão sem fio com mobilidade dos terminais.

A codificação wavelet foi proposta por Tzannes [1] como uma nova alternativa para superar os efeitos do desvanecimento. Esta técnica explora as propriedades de ortogonalidade das linhas da matriz de coeficientes wavelet $(\mathrm{MCW})$. O

Luiz G. de Q. Silveira Júnior e Francisco M. de Assis, Grupo de Estudos em Problemas de Otimização e Transmissão da Informação - GEPOTI, Departamento de Engenharia Elétrica, Universidade Federal de Campina Grande, Brasil, E-mails: junior@dee.ufcg.edu.br, fmarcos@dee.ufcg.edu.br.

Luiz F. de Q. Silveira, Centro Federal de Educação Tecnológica do Rio Grande do Norte, E-mail: lfelipe@cefetrn.br.

Ernesto L. Pinto, Laboratório de Comunicações Digitais, Departamento de Engenharia Elétrica, Instituto Militar de Engenharia - IME, Brasil, E-mail: ernesto@ime.eb.br. codificador wavelet multiplica, de forma sucessiva, os bits da fonte pelas linhas de uma MCW, espalhando a informação de cada bit sobre diversos intervalos de sinalização. Os símbolos produzidos em sua saída, denominados de símbolos wavelet, são multiníveis e não-eqüiprováveis.

O emprego da codificação wavelet em sistemas de transmissão sem fio requer o mapeamento da saída do codificador em símbolos de um esquema de modulação. Neste contexto, o uso de modulações com variação de amplitude é, em geral, de pouco interesse, por ser freqüente o emprego de amplificação não linear nos transmissores. Por outro lado, como as probabilidades a priori dos símbolos wavelet são muito diferentes, é necessário um criterioso projeto da constelação a ser usada, a fim de otimizar o desempenho do receptor (demodulador e decodificador wavelet).

Devido à ortogonalidade entre as linhas da MCW, a informação de cada bit pode ser recuperada no receptor de maneira simples, através de um banco de correlatores casados às linhas da matriz usada na codificação. $\mathrm{O}$ mecanismo de espalhamento da informação no tempo, antes da transmissão, e o recolhimento dela no receptor, contribui para melhorar a robustez do sistema de comunicação à combinação de efeitos do desvanecimento plano variante no tempo e de ruído localizado.

Um caminho novo para o desenvolvimento de receptores de elevado desempenho em diferentes tipos de canal começou a ser delineado a partir de 1993 com o advento dos esquemas de Codificação Turbo [2], [3], que usam concatenação de códigos separados por dispositivos de entrelaçamentos. No processo de decodificação, dois decodificadores do tipo "entrada e saída suaves" (SISO, de "sof-input soft-output") trocam informação de confiabilidade a respeito dos bits a serem decodificados. Num esquema turbo bem projetado a confiabilidade aumenta ao longo das iterações, até que seja produzida a saída final do decodificador.

Os resultados obtidos com os primeiros esquemas de codificação turbo foram rapidamente vistos como manifestações particulares de um princípio mais amplo (Princípio Turbo), segundo o qual ganhos significativos de desempenho, em sistemas com codificação de canal, podem ser obtidos processando iterativamente as amostras do sinal recebido, através de dois processadores com capacidade para trocar informações probabilísticas (em geral na forma de razões de verossimilhança logarítmicas) sobre as respectivas entradas.

Esta constatação deu origem à busca de diferentes formas de se explorar o princípio turbo, tanto em novas concatenações de códigos corretores de erro quanto em novas possibilidades de 
iteratividade envolvendo outro processador de recepção, como por exemplo um estimador de canal, um equalizador ou um dispositivo de sincronização [4].

O presente trabalho se situa nesta perspectiva, visando obter melhorias no desempenho de sistemas de transmissão digital, em canais com desvanecimento plano, através da exploração do princípio turbo em sistemas com codificação wavelet. Assim, propõe-se aqui um decodificador iterativo para uma concatenação serial que tem como codificador interno um codificador wavelet, tendo por ponto de partida o esquema de demodulação suave proposto em [5].

O desempenho do decodificador iterativo proposto é avaliado em um canal sujeito ao desvanecimento Rayleigh plano, via simulação computacional. Os resultados obtidos mostram que o decodificador wavelet SISO aqui apresentado é capaz de produzir informação extrínseca útil e atuar de maneira eficaz na decodificação iterativa. Em particular, foram observados ganhos de $2,5 \mathrm{~dB}$ na razão $E_{b} / N_{0}$ já a partir da primeira iteração, em relação a esquemas de recepção não iterativos anteriormente avaliados [5],[6],[7].

O restante deste artigo está organizado como segue. A Seção II apresenta uma revisão da técnica de codificação wavelet. A Seção III, é dedicada a apresentar os detalhes presentes na decodificação iterativa do sistema proposto, o qual utiliza a codificação wavelet em sequiências de blocos de bits da fonte que foram codificados convolucionalmente. Os resultados numéricos obtidos com a simulação do esquema proposto são mostrados e discutidos na Seção IV. Finalmente, a Seção V apresenta as conclusões deste trabalho.

\section{Fundamentos da Codificação Wavelet}

A codificação wavelet utiliza as linhas da matriz de coeficientes wavelets (MCW) com o objetivo de codificar os bits de informação. Uma MCW de ordem $m$ e gênero $g$ tem dimensão $m \times m g$, denotada por

$$
\mathbf{A}=\left(\begin{array}{ccc}
a_{0}^{0}, & \ldots, & a_{m g-1}^{0} \\
a_{0}^{1}, & \ldots, & a_{m g-1}^{1} \\
\vdots & & \vdots \\
a_{0}^{m-1}, & \ldots, & a_{m g-1}^{m-1}
\end{array}\right)
$$

cujas entradas podem pertencer ao conjunto dos números reais, ou complexos.

Em particular, uma matriz MCW é denominada real e plana, quando seus coeficientes são números reais escolhidos no conjunto $\{1,-1\}$, e satisfazem as seguintes condições [8]:

$$
\begin{aligned}
& \sum_{k=0}^{m g-1} a_{k}^{j}=m \sqrt{g} \delta_{0, j}, \quad 0 \leq j \leq m-1 \\
& \sum_{k} a_{k+m l}^{j} a_{k+m l^{\prime}}^{j^{\prime}}=m g \delta_{j, j^{\prime}} \delta_{l, l^{\prime}}, \quad 0 \leq j, j^{\prime} \leq m-1 \\
& 0 \leq l, l^{\prime} \leq g-1
\end{aligned}
$$

sendo $\delta_{j, j^{\prime}}$ o delta de Kronecker.

Apesar do algoritmo de codificação wavelet ser implementável com qualquer tipo de $\mathrm{MCW}$, este artigo considera apenas a codificação wavelet que utiliza MCWs inteiras e planas. A propriedade fundamental das $\mathrm{MCWs}$ para a codificação de canal é dada pela Equação 3, onde se vê que as linhas de uma MCW de ordem $m$ são mutuamente ortogonais sobre deslocamentos de comprimento $l m$, sendo $0 \leq l \leq g-1$. Além disso, vê-se na Equação 3 que cada linha é ortogonal a uma cópia de si mesma deslocada circularmente de $l m$, sendo $0<l \leq g-1$. Sob o ponto de vista da teoria de bancos de filtros digitais, as linhas $\left\{\mathbf{a}^{j}\right\}$ representam filtros passa-faixa de um banco de filtros com $m$-bandas [8].

O codificador wavelet originalmente proposto por Tzannes é ilustrado na Figura 1(a), onde a saída da fonte é a seqüência de bits $\left\{x_{n}\right\}$, com $x_{n} \in\{-1,+1\}$. Esta é inicialmente decimada em $m$ seqüências paralelas as quais são codificadas por bancos de filtros $\mathrm{WCM}_{\mathrm{j}}, j=\{0,1, \ldots, m-1\}$, resultando em $m$ sequiências paralelas de símbolos $\left\{y_{p m+q}^{j}, j=0,1, \ldots, m-\right.$ $1\}$, aqui denominados sub-símbolos wavelet. Em cada intervalo de tempo $n=p m+q, m$ sub-símbolos wavelet são adicionados de forma a gerar um símbolo wavelet $y_{n}$. A Figura 1(b) fornece detalhes da estrutura do bloco $\mathrm{WCM}_{\mathrm{j}}$, o qual é composto por $m$ filtros com resposta ao impulso finita, cada um com $g$ coeficientes pertencentes à $j$-ésima linha da MCW.

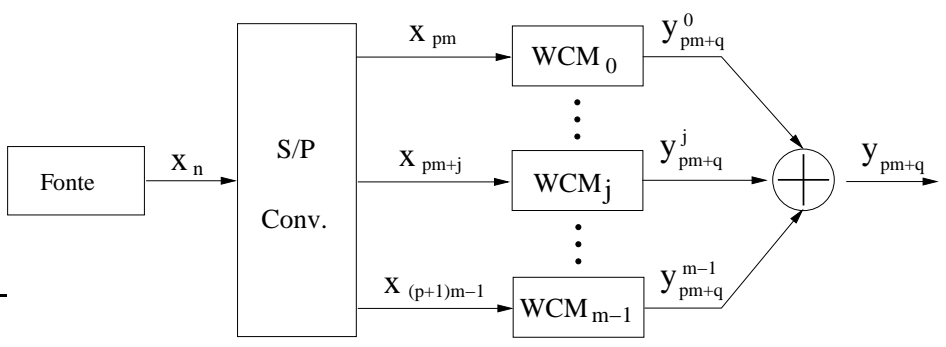

(a) Estrutura geral do codificador wavelet.

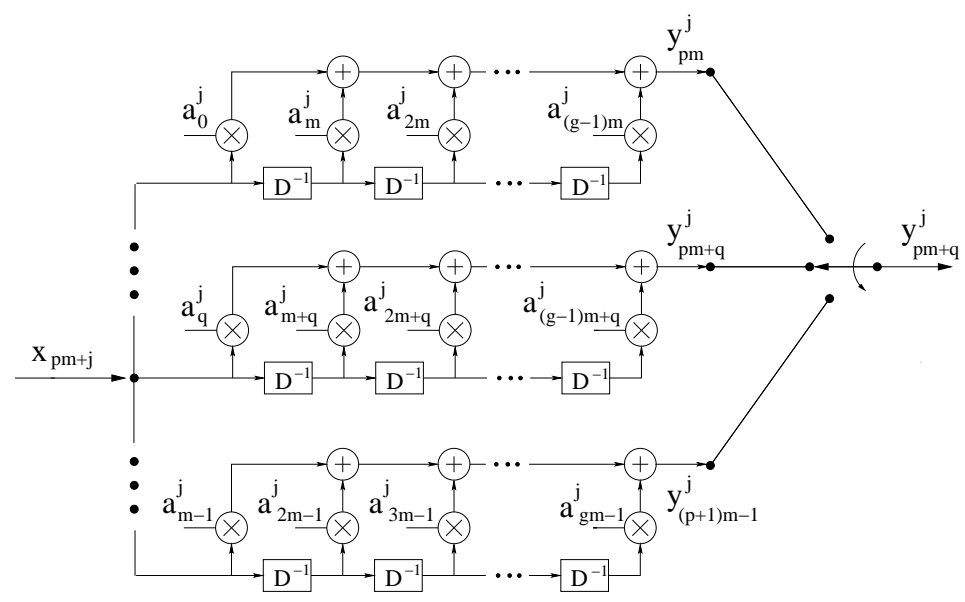

(b) Detalhamento do bloco $\mathrm{WCM}_{\mathrm{j}}$.

Fig. 1. Codificador wavelet formado por uma MCW de dimensão $m \times m g$. $\mathrm{O}$ bloco $\mathrm{WCM}_{\mathrm{j}}$ é especificado pela j-ésima linha da $\mathrm{MCW}$.

O símbolo wavelet gerado no intervalo de tempo $n=p m+q$ é dado por

$$
y_{p m+q}=\sum_{j=0}^{m-1} \sum_{l=0}^{g-1} a_{l m+q}^{j} x_{(p-l) m+j},
$$

e assume valores no conjunto $\mathcal{Y}=\{-m g,-m g+2, \ldots$, 
$-m g+2 k, \ldots, 0, \ldots, m g-2, m g\}$, de cardinalidade $m g+$ 1. Portanto, os símbolos wavelets são multiníveis e dependem de diversos bits de informação.

Como exemplo do processo de codificação com wavelets, a Tabela I ilustra o processo de formação dos símbolos wavelets quando uma MCW de dimensão $2 \times 8$ é usada. Esta tabela também ilustra a ocorrência de um período transitório de formação de símbolos wavelets, que neste caso se encerra em $n T_{s}=5$.

TABELA I

SÍMBOLOS GERADOS POR UMA MCW $2 \times 8$.

\begin{tabular}{|c|c|}
\hline$n T_{s}$ & $y_{n}$ \\
\hline 0 & $a_{0}^{0} x_{0}+a_{0}^{1} x_{1}$ \\
1 & $a_{1}^{0} x_{0}+a_{1}^{1} x_{1}$ \\
2 & $a_{2}^{0} x_{0}+a_{2}^{1} x_{1}+a_{0}^{0} x_{2}+a_{0}^{1} x_{3}$ \\
3 & $a_{3}^{0} x_{0}+a_{3}^{1} x_{1}+a_{1}^{0} x_{2}+a_{1}^{1} x_{3}$ \\
4 & $a_{4}^{0} x_{0}+a_{4}^{1} x_{1}+a_{2}^{0} x_{2}+a_{2}^{1} x_{3}+a_{0}^{0} x_{4}+a_{0}^{1} x_{5}$ \\
5 & $a_{5}^{0} x_{0}+a_{5}^{1} x_{1}+a_{3}^{0} x_{2}+a_{3}^{1} x_{3}+a_{1}^{0} x_{4}+a_{1}^{1} x_{5}$ \\
6 & $a_{6}^{0} x_{0}+a_{6}^{1} x_{1}+a_{4}^{0} x_{2}+a_{4}^{1} x_{3}+a_{2}^{0} x_{4}+a_{2}^{1} x_{5}+a_{0}^{0} x_{6}+a_{0}^{1} x_{7}$ \\
7 & $a_{7}^{0} x_{0}+a_{7}^{1} x_{1}+a_{5}^{0} x_{2}+a_{5}^{1} x_{3}+a_{3}^{0} x_{4}+a_{3}^{1} x_{5}+a_{1}^{0} x_{6}+a_{1}^{1} x_{7}$ \\
8 & $a_{6}^{0} x_{2}+a_{6}^{1} x_{3}+a_{4}^{0} x_{4}+a_{4}^{1} x_{5}+a_{2}^{0} x_{6}+a_{2}^{1} x_{7}+a_{0}^{0} x_{8}+a_{0}^{1} x_{9}$ \\
9 & $a_{7}^{0} x_{2}+a_{7}^{1} x_{3}+a_{5}^{0} x_{4}+a_{5}^{1} x_{5}+a_{3}^{0} x_{6}+a_{3}^{1} x_{7}+a_{1}^{0} x_{8}+a_{1}^{1} x_{9}$ \\
$\vdots$ & \\
\hline
\end{tabular}

\section{A. Decodificação Wavelet}

$\mathrm{Na}$ recepção, a seqüência de bits de informação $\left\{x_{n}\right\}$ pode ser recuperada a partir da seqüência recebida através da utilização de um banco de $m$ correlatores, cada um com comprimento igual a $m g$, casados às $m$ linhas da MCW. Assim, na ausência de erros, a saída do correlator $z^{j}, j \in$ $\{0,1, \ldots, m-1\}$, casado à linha $\mathbf{a}^{j}$ no tempo $i=m(g+p)-1$ é dado por

$$
\begin{aligned}
z_{i}^{j} & =\sum_{k=0}^{m g-1} a_{(m g-1)-k}^{j} y_{i-k} \\
& =\sum_{k=0}^{m g-1} \sum_{j^{\prime}=0}^{m-1} \sum_{l=0}^{g-1} a_{k}^{j}\left(a_{k-l m}^{j^{\prime}} x_{j^{\prime}+l m+i-(m g-1)}\right)
\end{aligned}
$$

Através da Equação (3), pode ser verificado que todos os termos do lado direito da Equação (5) se anulam, exceto aquele onde $j^{\prime}=j$ e $l=0$. Logo

$$
z_{i}^{j}=x_{j+i-(m g-1)} \sum_{k=0}^{m g-1} a_{k}^{j} a_{k}^{j}=m g x_{j+i-(m g-1)},
$$

sendo que $z_{i}^{j}$ pode ser utilizado para decidir sobre o bit $x_{j+i-(m g-1)}$ através da comparação com um limiar ajustado a zero, ou seja, o bit será -1 se $z_{i}=-m g$, ou, +1 se $z_{i}=+m g$. A simplicidade do processo de decodificação por correlação é uma das principais vantagens da técnica de codificação baseada em wavelets.

\section{DeCodificaÇÃo Iterativa PaRA CONCATENAÇÃo SERIAL COM CODIFICADOR WAVELET}

O modelo do sistema com decodificação iterativa e codificação wavelet proposto está ilustrado na Figura 2. Neste sistema, a saída da fonte gera uma sequiência de bits independentes e identicamente distribuídos (i.i.d.), pertencentes ao conjunto $\{-1,+1\}$. Essa sequiência de bits é particionada em blocos de $k$ bits, $\left\{\mathbf{x}_{k}\right\}$, os quais são codificados por um codificador convolucional, utilizado aqui como o codificador mais externo do sistema. Dessa forma, para cada bloco de $k$ bits, $\left\{\mathbf{x}_{k}\right\}$, o codificador convolucional gera um bloco de $n$ bits, $\left\{\mathbf{b}_{n}\right\}$, já incluídos os bits conhecidos de cabeçalho e de cauda.

Em seguida, os $n$ bits codificados de cada bloco $\left\{\mathbf{b}_{n}\right\}$ são embaralhados por um entrelaçador randômico e codificados por um codificador wavelet, o qual produz para cada bloco $\left\{\mathbf{b}_{n}\right\}$ em sua entrada, um bloco de $n$ símbolos wavelets, $\left\{y_{n}\right\}$, não-equiprováveis e correlacionados.

Os símbolos wavelets são modulados por sinais PSK, os quais possuem energia média unitária, e transmitidos por uma única antena sobre um canal com desvanecimento Rayleigh plano. O canal é admitido plano e essencialmente constante durante o intervalo de sinalização, apesar de ser variante no tempo.

A saída do canal, em tempo discreto, é modelada pela variável aleatória complexa $r_{n}=\alpha_{n} s_{n}+w_{n}$, sendo $\alpha_{n}$ a amostra de desvanecimento, $s_{n}$ o símbolo PSK transmitido, e $w_{n}$ o ruído complexo Gaussiano branco, de média nula e partes real e imaginária de mesma variância $N_{0} / 2$.

Deve ser observado que $n$ bits na entrada do codificador wavelet são codificados em $n$ símbolos wavelets, sendo enviados ao longo de $n$ intervalos de sinalização. Assim, a eficiência espectral de $1 \mathrm{bit} / \mathrm{s} / \mathrm{Hz}$ é facilmente obtida. O conjunto formado pelo codificador wavelet, modulador e demodulador, é modelado como o codificador interno do sistema que está sendo analisado.

Numa decodificação iterativa, o ganho de desempenho observado em relação aos sistemas com processamento de dados seqüencial, deve-se à troca de informação suave entre os dispositivos de decisão. No esquema aqui proposto, esta troca deve se dar entre o decodificador wavelet e o decodificador convolucional MAP iterativo baseado no algoritmo BCJR, que possui este nome devido às iniciais de seus autores (Bahl, Cocke, Jelinek e Raviv) [9]. Para isto, é necessário dispor de um decodificador wavelet do tipo SISO.

Propõe-se aqui um decodificador wavelet cuja entrada suave é obtida pela adoção de uma regra de decisão suave a ser utilizada no demodulador, derivada em [5]. Esta regra, que é baseada na minimização do erro médio quadrático entre o símbolo wavelet transmitido, $y_{i}$, e o estimado, $\tilde{y}_{n}$, é dada por

$$
\tilde{y}_{n}=\sum_{i=0}^{m g-1} y_{i} \frac{P\left(y_{i}\right) f_{R}\left(r \mid s_{i}\right)}{\sum_{j=0}^{m g-1} P\left(y_{j}\right) f_{R}\left(r \mid s_{j}\right)},
$$




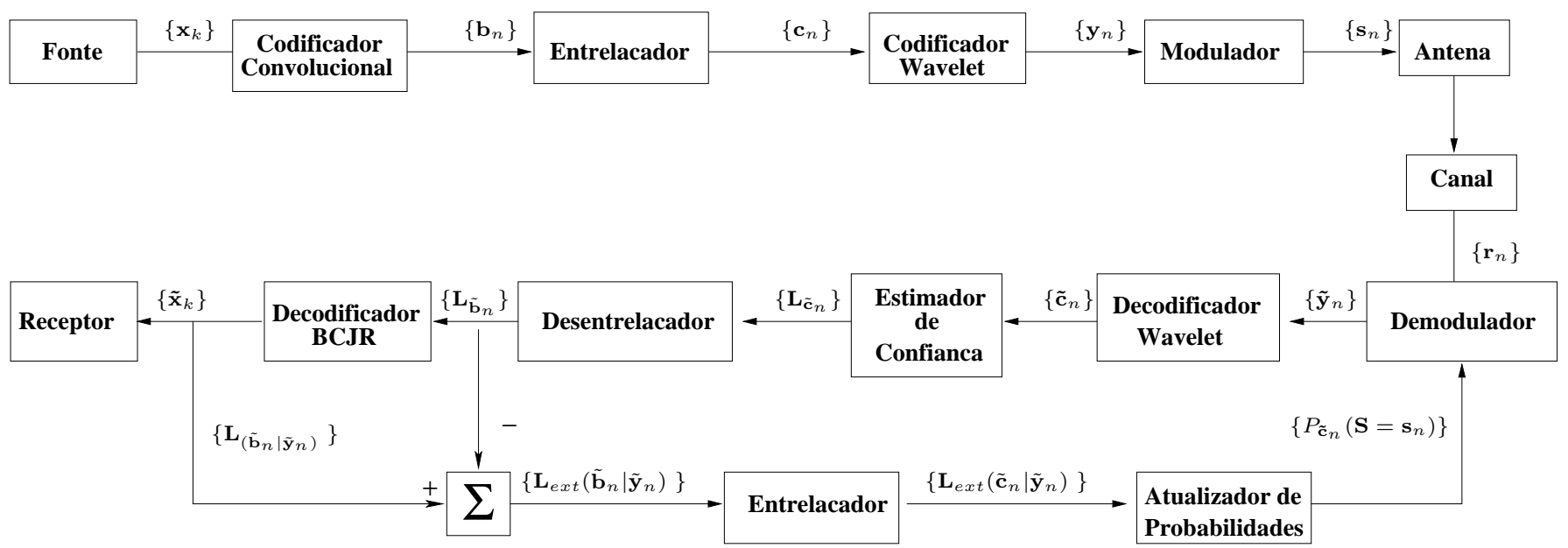

Fig. 2. Modelo do sistema com codificação wavelet e decodificação iterativa. O decodificador BCJR é utilizado para fornecer a informação extrínseca necessária para melhorar a confiabilidade dos bits de informação obtidos na recepção.

em que $s_{i}$ é o símbolo empregado pelo esquema de modulação para mapear o símbolo wavelet $y_{i}, P\left(Y_{i}=y_{i}\right)$ é a probabilidade a priori do símbolo wavelet e $f_{\mathbf{R}}\left(r \mid s_{i}\right)$ é a densidade condicional de probabilidade da variável aleatória que modela a saída do canal, $\mathbf{R}$.

É interessante observar que a regra expressa pela Equação (7) pode ser vista como uma regra de interpolação na qual os símbolos wavelets são convenientemente ponderados por suas respectivas probabilidades a posteriori. Para se tirar proveito do processamento iterativo, estas probabilidades devem ser atualizadas a cada iteração, usando a informação extrínseca proveniente do decodificador BCJR empregado.

Como este último fornece informações suaves chamadas de Razão de Log-Verossimilhança (LLR, Log-Likelihood Ratio), relativas aos bits de saída do codificador convolucional, introduziu-se o bloco Atualizador de Probabilidades para compatibilizar estas LLRs com as probabilidades $P\left(y_{i}\right)$ usadas pelo demodulador com saída suave. A análise deste bloco é realizada a seguir.

Deve-se observar inicialmente que a informação extrínseca fornecida pelo decodificador BCJR permite a imediata obtenção de probabilidades a priori dos bits de entrada do codificador wavelet.

Avaliando-se o processo de geração de símbolos wavelets por uma MCW de dimensão $2 \times 8$, conforme ilustrado pela Tabela I, observa-se que é possível expressar os símbolos wavelets como uma soma de bits de informação ponderados por coeficientes wavelets determinísticos.

Assim sendo, para se obter a probabilidade a priori do símbolo wavelet $Y_{i}=y_{i}$ deve-se inicialmente encontrar a probabilidade de ocorrência de cada bloco de bits que pode ser codificado no símbolo $Y_{i}=y_{i}$, e em seguida somar as probabilidades de todos esses blocos.

Portanto, de posse do vetor de probabilidades a priori dos bits codificados pelo codificador wavelet (vetor este obtido a partir das LLRs provenientes do decodificador BCJR), é possível calcular as probabilidades a priori dos símbolos wavelets, necessárias para a etapa de demodulação suave na recepção, conforme observado em (7).

Por outro lado, para se implementar o princípio turbo, é necessário fornecer ao decodificador BCJR uma informação extrínseca gerada pela saída suave, $\tilde{\mathbf{c}}_{k}$, do decodificador wavelet. Para tanto, admitiu-se que $\tilde{\mathbf{c}}_{k}$ segue uma distribuição Gaussiana condicionada ao bit transmitido, com média $\pm m g$ e variância $\sigma_{c}^{2}$, ou seja,

$$
P\left(\tilde{c}_{k} \mid c_{k}= \pm 1\right) \sim \mathcal{N}\left( \pm m g, \sigma_{c}^{2}\right)
$$

Então,

$$
p\left(\tilde{c}_{k} \mid c_{k}=-1\right)=\frac{1}{\sqrt{2 \pi \sigma_{c}^{2}}} \exp \left\{\frac{-1}{2 \sigma_{c}^{2}}\left(\tilde{c}_{k}+m g\right)^{2}\right\}
$$

e,

$$
p\left(\tilde{c}_{k} \mid c_{k}=+1\right)=\frac{1}{\sqrt{2 \pi \sigma_{c}^{2}}} \exp \left\{\frac{-1}{2 \sigma_{c}^{2}}\left(\tilde{c}_{k}-m g\right)^{2}\right\} .
$$

Dividindo-se (8) por (9) e tomando-se o logaritmo neperiano desta razão, obtém-se a métrica que estima a confiabilidade da decisão corrente, $\tilde{c}_{k}$, denotada por $L_{\tilde{c}_{k}}$,

$$
L_{\tilde{c}_{k}}=\frac{-2 m g}{\sigma_{c}^{2}} \cdot \tilde{c}_{k}
$$

Pode-se estimar a variância $\sigma_{c}^{2}$ de forma recursiva, a partir da estimativa da variância de cada um dos blocos de saída do decodificador wavelet. Pela Lei dos Grandes Números, essa estimativa converge probabilisticamente para o valor da variância da distribuição, à medida que o número de blocos, $\mathcal{G}$, ou o tamanho de cada bloco, $N$, aumenta. Assim, a partir de (10), pode-se estimar a confiabilidade sobre cada decisão do decodificador wavelet utilizando-se um procedimento recursivo para o cálculo da variância $\sigma_{c}^{2}$.

O cálculo recursivo desta variância, após a chegada do bloco $k+1$, pode ser expresso por 


$$
\hat{\sigma}_{c}^{2}(k+1)=\frac{1}{k+1}\left[k \cdot \hat{\sigma}_{c}^{2}(k)+\left(\frac{1}{N} \sum_{i=1}^{N} \tilde{c}_{k}^{2}(K \cdot N+i)-(m g)^{2}\right)\right]
$$

em que a estimativa $\hat{\sigma}_{c}^{2}(1)$ pode ser calculada após a saída do primeiro bloco do decodificador wavelet por $\hat{\sigma}_{c}^{2}(1)=$ $\frac{1}{N} \sum_{i=1}^{N} \tilde{c}_{k}^{2}(i)-(m g)^{2}$.

Deve ser observado que o método de decodificação iterativa proposto, utiliza o cálculo recursivo para estimar a variância da distribuição dos dados de saída do decodificador wavelet, utilizando os dados disponíveis em cada iteração. Logo, para um número L de iterações, existem L estimativas de variâncias, e L níveis de confiabilidade existentes sobre a qualidade das decisões realizadas pelo decodificador wavelet, ao menos até que ocorra a convergência destas.

\section{APLICAÇÃO}

Com o objetivo de avaliar o método proposto de decodificação iterativa, foram realizadas simulações computacionais do sistema exibido na Figura 2. Esta seção apresenta os resultados de desempenho obtidos em canais sujeitos ao desvanecimento Rayleigh plano. O sistema simulado utiliza um codificador convolucional com taxa igual a $1 / 2$ e uma MCW com dimensão $2 \times 8$. Para cada valor de relação sinal-ruído (RSR) simulado, foram avaliados uma quantidade mínima de $10^{3}$ erros para se estimar a respectiva taxa de erro de bit (TEB). Também foi admitido que o receptor tem conhecimento perfeito do estado do canal.

Como a técnica de codificação wavelet gera símbolos nãoequiprováveis, os ganhos de desempenho obtidos a partir destes sistemas são influenciados de forma significativa pelo esquema de modulação adotado. Assim, a Figura 3 exibe a constelação PSK obtida por busca computacional sub-ótima sobre o círculo unitário, utilizando o critério de minimização da taxa de erro de bit, presente em [10]. Nesta constelação, os sinais são associados aos valores dos correspondentes símbolos wavelet.

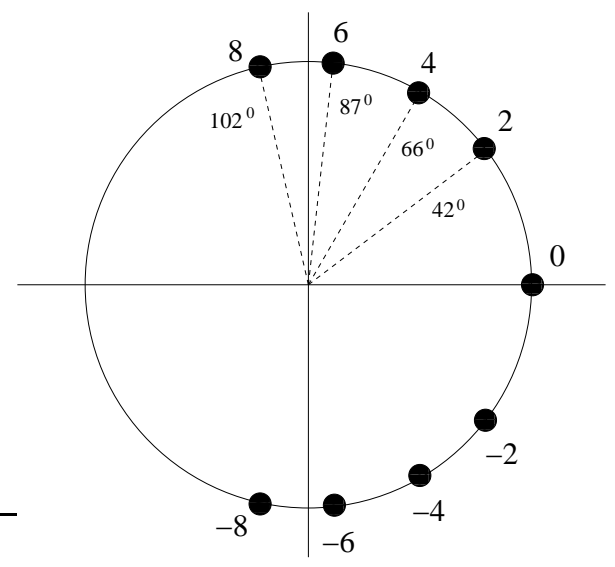

Fig. 3. Constelação PSK obtida para a MCW com dimensão $2 \times 8$.

A Figura 4 ilustra os resultados de desempenho obtidos com o método proposto para a decodificação iterativa em sistemas com codificação wavelet, apresentando-se a curva de TEB para diferentes tamanhos de bloco, na presença do desvanecimento Rayleigh plano.

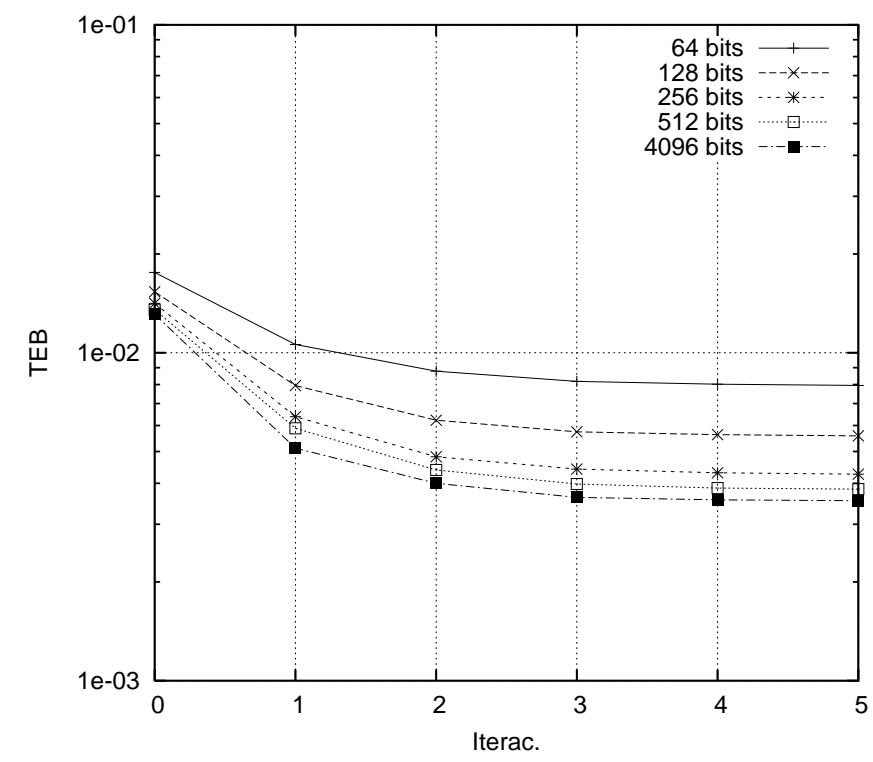

(a) Curva de TEB em função do número de iterações para diferentes tamanhos de blocos de bits transmitidos com RSR de 4dB.

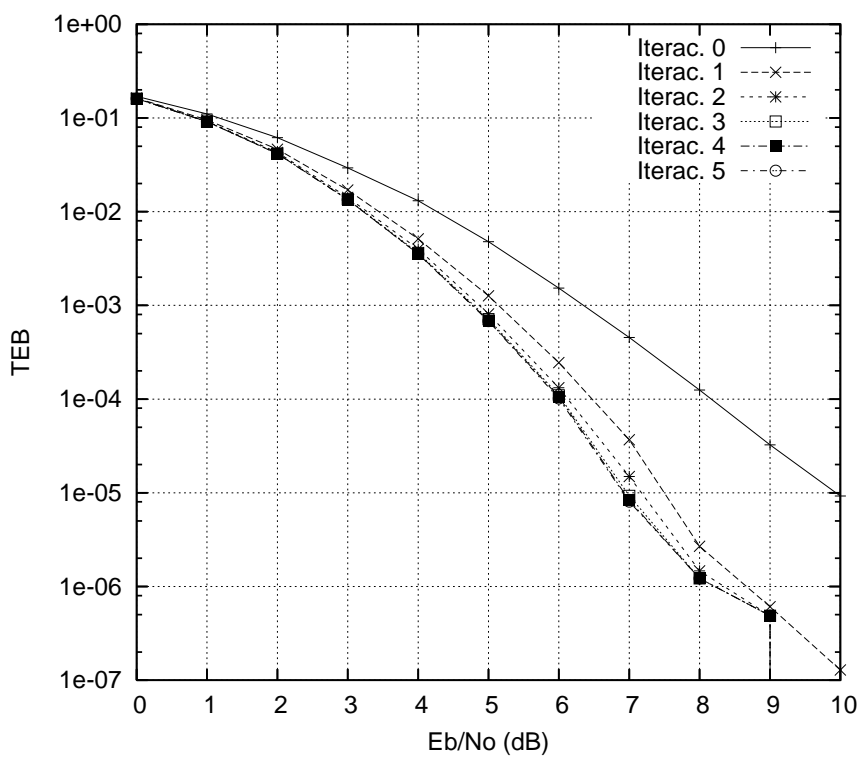

(b) Curva de TEB obtida com blocos de tamanho igual a 4096 bits.

Fig. 4. Curva de TEB do sistema com decodificação iterativa e codificação wavelet, para diferentes tamanhos de bloco e uma MCW de dimensão $2 \times 8$.

Na Figura 4(a) é mostrada a variação de TEB a cada iteração, para os cinco valores de tamanho de bloco testados $\left(64,128,256,512\right.$ e 4096), e para uma $\mathrm{RSR}=E_{b} / N_{0}=4 \mathrm{~dB}$. É possível observar que a TEB tende para um valor limite à medida que o tamanho do bloco transmitido é incrementado. Percebe-se também que os ganhos a cada iteração são maiores para tamanhos de bloco maiores.

A Figura 4(b) exibe a curva de desempenho do sistema proposto, para um tamanho de bloco igual a 4096 bits, com 
6 iterações. Através desta, é possível observar um ganho de desempenho em $E_{b} / N_{0}$ de $2,5 \mathrm{~dB}$, para valores de TEB abaixo de $10^{-5}$, já a partir da primeira iteração. Além disso, é possível verificar uma rápida convergência com o número de iterações.

Por outro lado, observou-se que o tamanho do bloco de símbolos é fundamental, ficando constatado que quanto maior for este, menor são os valores de TEB obtidos por iteração. Contudo, o aumento do tamanho de bloco é computacionalmente crítico, pois aumenta os requisitos de memória na recepção, para poder viabilizar o armazenamento dos valores de informação suave que são trocados entre os dispositivos de decisão, durante o processo iterativo.

\section{CONCLUSÃo}

Neste artigo, foi proposto um método para a decodificação iterativa para concatenação serial com codificador wavelet, sendo avaliado o seu desempenho sobre um canal sujeito ao desvanecimento Rayleigh plano.

Para este fim, foi necessário desenvolver um decodificador wavelet SISO. A partir do esquema de demodulação com saída suave proposto em [5], obteve-se um decodificador wavelet capaz de gerar informação extrínseca útil para operar iterativamente em parceria com um decodificador BCJR. No cálculo desta informação extrínseca, utilizou-se a variância dos dados de saída do decodificador wavelet, sendo esta estimada de forma recursiva a cada iteração.

Os resultados obtidos indicam que o uso desta estratégia de recepção constitui numa ferramenta eficaz para promover o aumento do ganho de desempenho de sistemas com codificação wavelet, sujeitos ao desvanecimento Rayleigh plano.

No futuro, os autores pretendem investigar outras aplicações da técnica de decodificação iterativa aqui proposta.

\section{AGRADECIMENTOS}

Os autores gostariam de expressar seus agradecimentos à CAPES e ao CNPq pelo apoio financeiro ao trabalho.

\section{REFERÊNCIAS}

[1] M. A. Tzannes and M. C. Tzannes, Bit-by-bit channel coding using wavelets, Proceedings of IEEE GLOBECOM'92, pp. 684-688, April, 1992.

[2] C. Berrou, A. Glavieux and P. Thitimajshima. Near Shannon limit error-correcting coding and decoding: Turbo-Codes. in Proceedings of the 1993 Communication Conference, ICC'93, Geneva, Switzerland, pp.1064-1070, May-1993.

[3] C. Berrou, The ten-year-old turbo codes are entering into service, IEEE Communications Magazine, vol. 41, no. 8, pp. 110-116, Aug. 2003.

[4] M. Tüchler, R. Koetter and A. Singer, Turbo equalization, IEEE Signal Processing Magazine, vol. 21, no. 1, pp 67-80, Jan. 2004.

[5] L. G. Q. Silveira Júnior, L. F. Q. Silveira, E.L. Pinto e F.M. de Assis. Soft-Decision Demodulation of Wavelet-Coded PSK Signals over Flat Rayleigh Fading Channels. ITS'2006 - VI International Telecommunications Symposium. September 3-6, 2006, Fortaleza-CE, Brazil.

[6] L. F. Q. Silveira and F. M. Assis and Ernesto L. Pinto. Performance of a PSK System with Wavelet Time Diversity over Flat Rayleigh Fading Channels. Proceedings of International Microwave and Optoelectronics Conference, IMOC'2003. 2003.

[7] L. F. Q. Silveira, F. M. Assis and E. L. Pinto, A New Wavelet Coded PSK System over Time-varying Flat Fading Channels, Fifth IEEE Workshop on Signal Processing Advances in Wireless Communications - SPAWC'2004, July 2004.
[8] H. L. Resnikoff and R. O. Wells Jr., Wavelet Analysis: The Scalable Structure of Information, Springer-Verlang, ISBN 0-387-98383-X, 1998

[9] L. R. Bahl, J. Cocke, F. Jelinek, and J. Raviv, Optimal Decoding of Linear Codes for Minimizing Symbol Error Rate, IEEE Trans. Inform. Theory, vol. 20, pp 284-287, Mar. 1974.

[10] L. F. Q. Silveira, Wavelet-Coded System Analysis over Time-varying Flat Fading Channels (in Portuguese), Ph.D. Thesis/DEE-UFCG, junho de 2006. 